\title{
Etude de l'aptitude à germer in vitro du pollen de colza (Brassica napus L.) récolté par l'abeille domestique (Apis mellifica L.)
}

\author{
J. Mesquida ${ }^{1}$ et M. Renard 2
}

\author{
1 Laboratoire de recherches de la chaire de zoologie, Domaine de la Motte-au-Vicomte, BP 29, \\ 35650 Le Rheu; \\ 2 Station d'amélioration des plantes, Demaine de la Motte au Vicomte, BP 29, 35650 Le Rheu, \\ France
}

(reçu le 10 mars 1988, accepté le 8 mars 1989)

\begin{abstract}
Résumé - Les auteurs ont comparé l'aptitude à germer in vitro du pollen de colza prélevé à la main sur les fleurs (témoin) à celui récolté par l'abeille domestique. Des résultats obtenus, il ressort que, par rapport au témoin, le pollen prélevé sur les abeilles (tête, thorax, pelotes) a manifesté une baisse significative de son pouvoir germinatif (20 à $45 \%$ immédiatement après le butinage, 60 à $85 \% 2 \mathrm{~h}$ après). Son activité germinative a cessé en $48 \mathrm{~h} . \mathrm{A}+7^{\circ} \mathrm{C}$, le taux de germination du pollen de pelotes s'est maintenu autour de $35 \%$ (contre $70 \%$ pendant $72 \mathrm{~h}$ pour le témoin), et son activité germinative a cessé en $96 \mathrm{~h}$, contre 264 h environ pour le témoin. Cette perte de l'aptitude à germer in vitro du pollen de colza récolté par l'abeille semble correspondre à l'effet inhibiteur de leurs sécrétions glandulaires, déjà signalé sur du pollen d'autres espèces végétales.
\end{abstract}

pollen - Brassica napus - germination in vitro - Inhibition - Apls mellfica

Summary - In vitro germination ability of rapeseed (Brassica napus L.) pollen collected by honey bees (Apls mellifera L.). Germination percentage of oilseed rape pollen taken from foraging honey bee was compared with that of pollen hand-collected directly from flowers (1984 and 1985). The aim of the study was to assess the decrease of germination ability and to know whether it could be a factor limiting the pollination, mainly in the case of F1 hybrid seed production. The trials concerned : the germination of pollen from bee heads, thoraces and pellets (trial I); the germination of pollen with respect to the time of presence on the thoraces of honey bees (trial II); the germination of pollen pellets stored at various temperatures (trial III). Germination percentage of pollen collected by bees was significantly lower than for pollen taken directly from flowers (control) : it decreased from 20 to $45 \%$ just after collection and from 60 to $85 \% 2 \mathrm{~h}$ later. The difference was greater for pollen from heads and pollen pellets than for pollen from thoraces (Table 1). Forty-eight hours after collection, bee pollen germination percentage was virtually zero (Fig. 1). Germination percentage of pollen trom pellets varied appreciably with conservation temperature (Fig. 2) : at $+7^{\circ} \mathrm{C}$ it was maintained at $35 \%$ over $48 \mathrm{~h}$ (70\% over $72 \mathrm{~h}$ for controls) and was zero after $96 \mathrm{~h}$, as compared with $264 \mathrm{~h}$ for controls. At $+15^{\circ} \mathrm{C}$ germination percentage was only $10 \%$ over $48 \mathrm{~h}(70 \%$ over $24 \mathrm{~h}$ and $30 \%$ over $48 \mathrm{~h}$ for controls) and zero at $72 \mathrm{~h}$ for pollen from pellets and controls. At extreme temperature $\left(-25^{\circ} \mathrm{C}\right.$ and $\left.+30^{\circ} \mathrm{C}\right)$ germination percentage was zero under the experimental conditions. The decrease in in vitro germination percentage of pollen collected by honey bees may 
be attributed to inhibitory effects of bee glandular secretions. The results do not allow to conclude about the possible factor limiting the pollination; they have to be proved by studies of in vivo germination.

pollen - Brassica napus - in vitro germination - inhibition - Apls mellifera

Zusammenfassung - Studien über dle in vitro Kelmfähigkeit des von der Honigbiene gesammelten Rapspollens (Brassica napus L.). In den Jahren 1984 und 1985 wurde die Fähigkeit zur in vitro Keimung von Rapspollen (Brassica napus $L$.) bestimmt, und zwar im Vergleich zwischen handgesammeltem Pollen und von der Honigbiene (Apis mellifera L.) gesammeltem Pollen. Das Ziel der Untersuchung war, festzustellen, ob der Verlust der Keimfähigkeit durch die Bienenbestäubung hervorgerufen wird, oder eine Folge der Produktion von Hybridsamen F1 ist. Die Methoden und Techniken der in vitro-Keimung wurden bereits anderweitig genau beschrieben (Mesquida et al., 1987). Die Untersuchung umfaßte folgende Teile : Keimfähigkeit von Pollen des 1. Tages, gesammelt auf verschiedenen Teilen des Körpers der Honigbiene (Kopt, Brustteil und Pollen-höschen) (Versuch l); Keimfähigkeit von Pollen in Abhängigkeit von der Dauer des Verweilens auf dem Brustteil der Biene (Versuch II); Keimfähigkeit von Pollen aus Pollenhöschen, die bei verschiedenen Temperaturen konserviert waren (Versuch III). Die Ergebnisse dieser Versuche zeigten eine klare Minderung der Keimfähigkeit von Pollen, der durch Bienen gesammelt wurde (PoAb) gegenüber Pollen, der von Hand auf den Blüten gesammelt wurde (Kontrolle) : von 20 bis $45 \%$ unmittelbar nach der Ernte und von 60 bis 85\% 2 Stunden später. Der Rückgang ist besonders groß bei den PoAb vom Kopf und aus den Pollenhöschen gegenüber dem des Thorax (Tabelle I). Nach ca. $48 \mathrm{~h}$ hat der Pollen seine gesamte Keimfähigkeit verioren (Fig. 1). Die Keimfähigkeit des Pollens aus Pollenhöschen (PoPel) variiert stark mit der Temperatur, bei der er gelagert wird (Fig. 2) : bei $+7^{\circ} \mathrm{C}$ hält sich die Keimfähigkeit auf 35\% während $48 \mathrm{~h}$ (gegenüber $70 \%$ über $72 \mathrm{~h}$ bei der Kontrolle) und erreicht $0 \%$ nach $96 \mathrm{~h}$ (gegenüber $264 h$ bei der Kontrolle); bei $+15^{\circ} \mathrm{C}$ waren nach $24 h$ und nach $48 h$ nur noch $10 \%$ keimfähig (gegenüber $70 \%$ nach $24 \mathrm{~h}$ und $30 \%$ nach $48 \mathrm{~h}$ bei der Kontrolle), gar keine Keimfähigkeit zeigt sich mehr nach $72 \mathrm{~h}$ sowohl für die PoPel wie für die Kontrolle; bei extremen Temperaturen $\left(-25^{\circ} \mathrm{C}\right.$ und $\left.+30^{\circ} \mathrm{C}\right)$ zeigten sowohl die PoPel wie die Kontrolle keinerlei Keimfähigkeit mehr.

Die vorliegende Abeit bestätigt die inhibitorische Aktion der Bienen auf die Keimfăhigkeit des Pollens in vitro, die auch schon von zahlreichen anderen Autoren mit anderen Pflanzenarten nachgewiesen wurde. Die Resultate erlauben jedoch nicht, Aussagen über die Faktoren zu machen, die die Bestäubung limitieren; diese sollten im Zuge von Untersuchungen der Keimfähigkeit in vivo gemacht werden.

Pollen - Brassica napus - in vitro Kelmfähigkelt — Inhibition - Apls mellifera

\section{Introduction}

Dans une étude précédente, nous avons montré que l'aptitude à la germination in vitro du pollen de colza (Brassica napus L.) est de courte durée, de l'ordre de $72 \mathrm{~h}$ (Mesquida et al., 1987). Dans la présente note, nous nous sommes intéressés à l'étude comparée de la germination in vitro du pollen de colza prélevé à la main sur les fleurs et à celle du pollen récolté par les abeilles domestiques (Apis mellifica L.).
Sur d'autres plantes cultivées (Betula sp.; Papaver sp.; Petunia sp.), des recherches effectuées par Maurizio (1960) ont permis de montrer que les sécrétions acides $(\mathrm{pH}=4,8)$ des glandes hypopharyngiennes des abeilles exercent une action inhibitrice non négligeable sur le pouvoir germinatif du pollen frais récolté à la main. Par la suite, Lukoschus et Keularts (1968) ont localisé la substance inhibitrice de la germination du pollen de pelotes d'abeilles dans la glande mandibulaire. Elle a été identifiée comme étant 
l'acide 10-hydroxy-2-décénoïque (acide gras contenu dans la gelée royale).

En 1962, sur un certain nombre de plantes cultivées, dont des choux (Brassica oleracea $L$.), Kraai a trouvé que les abeilles ayant séjourné $12 \mathrm{~h}$ dans leur ruche après leur butinage ne sont plus porteuses sur leur corps de pollen apte à germer in vitro.

Cependant, Lespinasse et Chevreau (1984), en utilisant un gène marqueur chez le pommier (Malus sp.), tendent à prouver qu'il reste encore sur le corps des abeilles du pollen capable de germer in vivo après un séjour de la ruche de $48 \mathrm{~h}$ en chambre froide $\left(+5^{\circ} \mathrm{C}\right)$. Hoopingarner et al. (1984) ont observé de leur côté que les abeilles étaient susceptibles d'échanger du pollen viable dans la ruche et ainsi de provoquer des pollinisations croisées chez le pommier. Pour Okada et al. (1983), le pollen de pelotes d'abeilles présente une certaine quantité de pollen apte à germer encore au $3^{\theta}$ et au $4^{\theta}$ jour de sa récolte chez le poirier (Pirus sp.), le pommier et le pêcher (Prunus persica). Mais, les premiers effets d'inhibition dus aux abeilles se manifestent clairement dès le

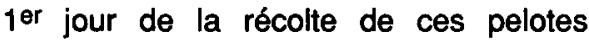
(Johansen, 1955; Okada et al., 1983). En revanche, le stockage au froid des pelotes de pollen ou du pollen récolté à la main permet de maintenir plus longtemps la faculté germinative (Kremer, 1949; Singh et Boynton, 1949; Griggs et Vansell, 1949; Firman, 1980; Okada et al., 1981; Filiti et Montalti, 1982; Klug, 1984; Cerceau-Larrival et Challe, 1986).

L'objectif de ce travail est de tenter de vérifier l'effet inhibiteur que les abeilles exercent sur l'aptitude à germer du pollen de colza qu'elles récoltent ou qui se dépose sur différentes parties du corps : tête et thorax notamment. II s'agit de savoir si cet effet inhibiteur peut être un facteur limitant de la pollinisation, en particulier dans les dispositifs faisant alterner au champ des plantes "mâles" donneuses de pollen et des plantes «femelles" (mâles stériles) receveuses de pollen pour la production de semences hybrides F1.

Nous présentons, dans cet article, les résultats obtenus en 1984 et 1985.

\section{Matériel et Méthodes}

Les échantillons d'abeilles de chaque essai, porteuses de pelotes de pollen sur leurs pattes postérieures, ont été prélevés au champ pendant leur butinage en même temps que les échantillons de fleurs de colza d'hiver (var. « Jet neuf») et de printemps (var. «Brutor»), au stade de la déhiscence des anthères, pour constituer les lots témoins. Ces prélèvements ont été effectués généralement entre $12 \mathrm{~h}$ et $14 \mathrm{~h}$ GMT, c'est-à-dire au moment où se situe, d'après Williams (1984), le pic de production de pollen dans la nature.

Mais, bien que le pollen de pelotes d'abeilles ne participe pas directement à la pollinisation pendant le butinage, l'étude a cependant été étendue à celui-ci. Nous supposons que ce pollen est identique à celui qu'elles portent sur leur corps et qu'il a été, en tout cas, récolté au même moment sur les mémes fleurs.

Après les prélèvements, les abeilles porteuses de pelotes de pollen ont toutes été anesthésiées au gaz carbonique $\left(\mathrm{CO}_{2}\right)$ pendant quelques minutes dans des sacs en plastique. Elles ont été disposées ensuite dans des boîtes de Petri fermées et exposées, avant les mises en germination du pollen de leurs pelotes, à la température ambiante du laboratoire, de l'ordre de $18^{\circ} \mathrm{C}$. Nous avons fait subir ce même traitement aux échantillons de fleurs qui ont servi de témoin.

Les essais de germination in vitro ont été effectués sur le milieu alcalin $(\mathrm{pH}=8,5$ ) proposé par Roberts et al. (1983). Les taux de germination sont estimés après $24 \mathrm{~h}$ d'incubation du pollen en étuve à $25^{\circ} \mathrm{C}$ en appliquant les techniques de germination in vitro (méthode de la goutte pendante), les méthodes d'observations et d'évaluation des taux de germination déjà décrites par Mesquida et al. (1987). 
Nous avons réalisé 3 types d'essais :

- I : essai de germination à 6 répétitions effec-

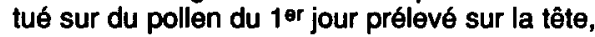
le thorax et les pelotes des abeilles, ainsi que sur les fleurs (témoin) à 2 moments de la journée : l'un immédiatement après les prélèvements des échantillons au champ (J0); l'autre 2 $\mathrm{h}$ après les prélèvements $(\mathrm{JO}+2 \mathrm{~h})$;

- II : essai de germination à 3 répétitions effectué le même jour ( 17 mai à $12 \mathrm{~h}$ ) sur du pollen prélevé sur le thorax des abeilles et sur les fleurs (témoin) pendant 4 jours consécutifs (du 14 au 17 mai) à la même heure (12 h) et disposés en boîtes de Petri fermées à la température ambiante du laboratoire. Un échantillon supplémentaire d'abeilles et de fleurs a été prélevé le $4^{e}$ jour (17 mai) à $9 \mathrm{~h}$. Les échantillons ainsi prélevés ont permis d'obtenir du pollen, dont la durée de présence sur le corps des abeilles était de : quelques minutes pour la récolte du 17 mai à $12 \mathrm{~h}$ (JO), $3 \mathrm{~h}$ pour la récolte du 17 mai à $9 \mathrm{~h}, 24 \mathrm{~h}$ pour la récolte du 16 mai, 48 h pour la récolte du 15 mai, 72 h pour la récolte du 14 mai;

- III : pour effectuer cet essai, tous les échantillons d'abeilles et de fleurs ont été prélevés au champ le même jour et à la même heure. Ils ont été ensuite placés, en boîtes de Petri fermées, dans 4 conditions fixes de température : $-25^{\circ} \mathrm{C} ;+7^{\circ} \mathrm{C} ;+15^{\circ} \mathrm{C}$ et $+30^{\circ} \mathrm{C}$.

Les mises en germination in vitro, essais a 3 répétitions, du pollen de pelotes et de fleurs (témoin) ont été effectuées chaque jour à la même heure (12 h) pendant 12 jours consécutifs. GMT.

Toutes les heures sont données en heure

Les comparaisons des moyennes des taux de germination ont été effectúés avec le test non paramétrique $\mathrm{H}$ de Kruskall et Wallis (1952).

\section{Résultats}

Faculté germinative du pollen du 1er jour récolté par les abeilles (essai l)

Le pouvoir germinatif du pollen récolté par les abeilles diminue assez rapidement par rapport à celui du témoin (Tableau I).

Immédiatement après le butinage (à Jo), les différences apparaissent déjà significatives (Tableau I). Le pollen de la tête et celui des pelotes prélevés sur les abeilles présentent les taux les plus bas. Ils correspondent à près de la moitié (de l'ordre de 55\%) du taux moyen du témoin.

Tableau I. Taux moyens de germination du pollen prélevé sur des fleurs de colza ou récolté par les abeilles à $\mathrm{JO}$ et $\mathrm{JO}+2 \mathrm{~h}$ (essai $\mathrm{l}$ ).

\begin{tabular}{|c|c|c|c|c|c|}
\hline & \multicolumn{3}{|c|}{ A - Abeilles } & \multirow{2}{*}{$\begin{array}{c}\text { B - Flours } \\
4\end{array}$} & \multirow[t]{2}{*}{ Différence } \\
\hline & 1 & 2 & 3 & & \\
\hline jo & $\begin{array}{c}30,13(c) \\
( \pm 1,02)\end{array}$ & $\begin{array}{c}44,46(a-b) \\
( \pm 4,21)\end{array}$ & $\begin{array}{c}30,65(b-c) \\
\quad( \pm 4,83)\end{array}$ & $\begin{array}{c}54,16(a) \\
( \pm 3,67)\end{array}$ & $\star$ \\
\hline $\mathrm{JO}+2 \mathrm{~h}$ & $\begin{array}{c}19,25(e-c) \\
( \pm 5,97)\end{array}$ & $\begin{array}{c}21,93(\mathrm{e}) \\
( \pm 2,31)\end{array}$ & $\begin{array}{l}7,81(d) \\
( \pm 1,26)\end{array}$ & $\begin{array}{c}54,92(a) \\
( \pm 2,32)\end{array}$ & * \\
\hline Différences & NS & $*$ & * & NS & \\
\hline
\end{tabular}

Les moyennes sont données avec l'erreur type $( \pm s / \sqrt{n}): n=6$. Les moyennes suivies d'une même lettre ne sont pas significativement différentes. 1 : pollen de la tête; 2 : pollen du thorax; 3 : pollen de pelotes; 4 : témoin. NS : differences non significatives; ${ }^{\star}$ : differences significatives à $5 \%$. 
De son côté, le pollen du thorax accuse aussi un taux de germination inférieur à celui du témoin $(81 \%)$, mais la différence n'est pas significative.

A $2 \mathrm{~h}$ du butinage, le Tableau I montre que l'aptitude à germer du pollen récolté par les abeilles baisse significativement, alors qu'elle apparaît plus stable pour le pollen de fleurs. La diminution du taux de germination semble cependant plus rapide dans le cas du thorax (de l'ordre de $22 \%$ contre $45 \%$, soit une perte de $50 \%$ environ) et des pelotes (de l'ordre de $8 \%$ contre $31 \%$, soit une perte de $75 \%$ environ). Le taux de germination du pollen de la tête n'a diminué que de $36 \%$ seulement.

Evolution dans le temps de la faculté germinative du pollen récolté par les abeilles (essai II)

La Figure 1 montre que le pollen récolté par les abeilles (pollen du thorax) et exposé. à la température ambiante du laboratoire perd son aptitude à germer dans un temps très court.

Au début du butinage, (à J0), le pollen récolté par les abeilles subit, comme cela a été démontré précédemment (Tableau I), une diminution importante et significative du taux moyen de germination par rapport à celui du témoin $(50 \%$ contre $80 \%$ pour le témoin). II régresse ensuite, plus rapidement d'ailleurs que le témoin, à $3 \mathrm{~h}$ du butinage (de l'ordre de $14 \%$ contre $67 \%$ pour le témoin, soit une perte de $79 \%$ ). Entre $3 \mathrm{~h}$ et $24 \mathrm{~h}$, le pollen récolté par les abeilles semble perdre moins vite son pouvoir germinatif qu'entre $24 \mathrm{~h}$ et $48 \mathrm{~h}$. Mais la quantité de pollen qui demeure apte à germer à $48 \mathrm{~h}$ est tout à fait négligeable (de l'ordre de $0,7 \%$ contre $32 \%$ pour le témoin). Après $48 \mathrm{~h}$, la faculté germinative est pratiquement nulle.
Influence de la température sur la faculté germinative du pollen de pelotes (essai III)

La Figure 2 fait apparaître des variations importantes de l'évolution de l'aptitude à germer du pollen de pelotes en fonction des températures de conservation.

A $7{ }^{\circ} \mathrm{C}$, le pollen de pelotes qui semble accuser une régression brutale dès le

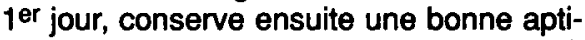
tude à germer pendant 2 jours (à $24 \mathrm{~h}$ et à $48 \mathrm{~h}$, le taux moyen étant de l'ordre de $35 \%$, Fig. 2A). Le taux de germination régresse cependant brusquement à $72 \mathrm{~h}$ (de l'ordre de $2 \%$ ) et cesse toute activité de germination à partir de $96 \mathrm{~h}$. Le pollen de fleurs (témoin, Fig. 2B) maintient, en revanche, une certaine aptitude à germer pendant $264 \mathrm{~h}$ (11 jours) : les taux moyens de germination, qui sont de l'ordre de $70 \%$ pendant $72 \mathrm{~h}$, diminuent rapidement ensuite (de l'ordre de 10 à $15 \%$ entre $96 \mathrm{~h}$ et $168 \mathrm{~h}$ et de $1 \%$ à $264 \mathrm{~h})$, et toute activité de germination cesse à $288 \mathrm{~h}$ (12 jours).

A $15^{\circ} \mathrm{C}$, les taux de germination sont peu élevés à $24 \mathrm{~h}$ et à $48 \mathrm{~h}$ (de l'ordre de $10 \%$ ) et nuls à $72 \mathrm{~h}$ pour le pollen de pelotes (Fig. 2A), tandis que la courbe obtenue avec le pollen de fleurs fait appa-

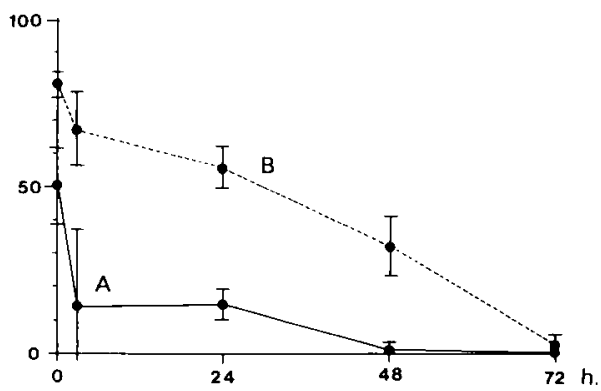

Fig. 1. Evolution de l'aptitude à germer du pollen prélevé sur des fleurs de colza et sur le thorax des abeilles (essai II). En abscisse : áge du pollen (en h); en ordonnée : pourcentage de pollen germé. Moyennes et intervalles de confiance : $A$ : pollen prélevé sur le thorax des abeilles; B : pollen de fleurs (témoin). 
raître un bon taux de germination jusqu'à $48 \mathrm{~h}$. Ce pollen ne germe plus à $72 \mathrm{~h}$ (Fig. 2B).

Aux températures extrêmes $\left(-25^{\circ} \mathrm{C}\right.$ et $\left.+30^{\circ} \mathrm{C}\right)$, le pollen d'abeilles et de fleurs n'expriment aucune aptitude à germer dès le $2^{\theta}$ jour (à 24 h) (Fig. $2 A$ et B).

\section{Discussion et Conclusion}

Le présent travail apporte une nouvelle preuve de l'action inhibitrice que les
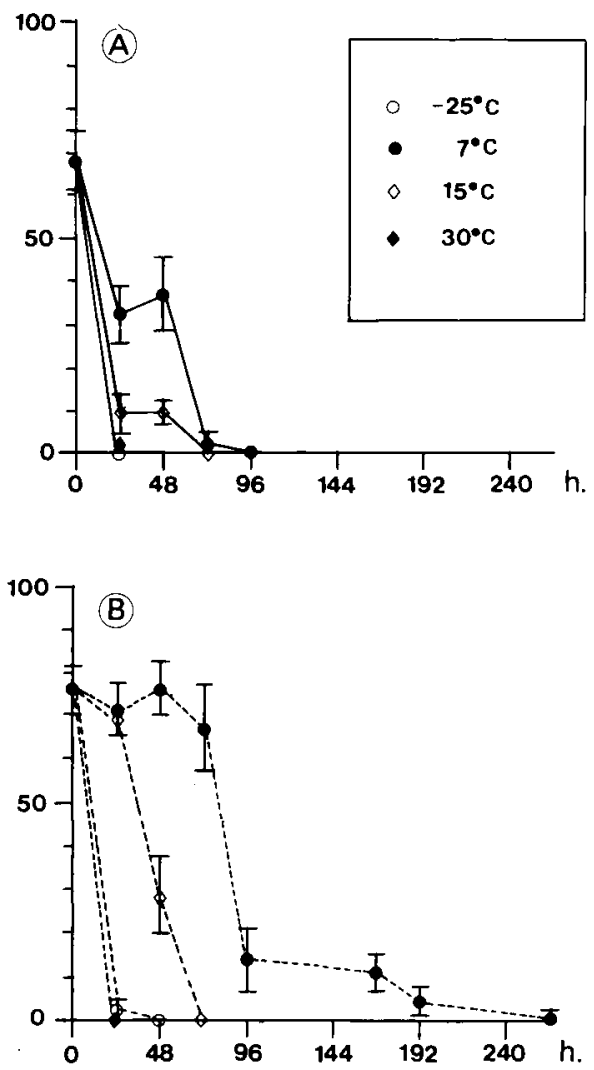

Flg. 2. Influence de la température sur la faculté germinative du pollen prélevé sur des fleurs de colza et sur des pelotes (essai III). Même légende que Figure 1. A. Pollen de pelotes d'abeilles. B. Pollen de fleurs (témoin). abeilles exercent sur la germination in vitro du pollen et confirme les travaux de nombreux auteurs (plus particulièrement ceux de : Singh et Boynton, 1949; Johansen, 1955; Maurizio, 1960; Lavie, 1960; Lukoschus et Keularts, 1968; Okada et al., 1983; Klug, 1984), qui ont observé le phénomène sur du pollen d'autres espèces végétales.

Le présent travail démontre aussi que cette perte progressive de la faculté germinative du pollen de colza semble se produire plus ou moins vite selon la partie du corps de l'abeille sur laquelle il est fixé et aussi selon les conditions de conservation.

Variations selon les parties du corps des abeilles

Dans nos conditions d'expérimentation, on constate tout d'abord que le pollen fixé sur la tête, dont on pense qu'il peut être plus rapidement en contact avec les substances inhibitrices, perd plus vite sa faculté germinative que celui fixé sur le thorax. Mais un équilibre semble s'établir ensuite où, à $2 \mathrm{~h}$ de la récolte, on observe une perte équivalente de l'aptitude à germer du pollen de la tête avec celle du thorax. En revanche, la perte de la faculté germinative du pollen de pelotes est plus importante que celle du pollen fixé sur le corps. En moins de $2 \mathrm{~h}$, on enregistre une perte du pouvoir germinatif du pollen de pelotes qui est de l'ordre de $85 \%$ par rapport au pollen de fleurs, alors que, dans le même temps, celle du pollen du corps n'est que de l'ordre de $60 \%$.

La perte de la faculté germinative du pollen de pelotes qui apparaît plus rapide que celle du pollen logé sur le corps des abeilles semble corroborer l'hypothèse de Maurizio (1968). Selon cet auteur, ce pollen, plus humecté par l'abeille au moment 
de la confection et de la fixation des pelotes sur ses pattes postérieures recevrait, par conséquent, plus de substances inhibitrices que celui situé sur le corps, ce qui expliquerait la différence observée. L'action inhibitrice serait donc fortement influencée par la quantité de substances actives émises par l'insecte lui-même, ce qui paraît logique, encore que cette hypothèse demanderait à être vérifiée.

\section{Variations selon les conditions de conservation}

L'efficacité des substances inhibitrices et la vitesse de leur action sur le pollen récolté par l'abeille peuvent aussi varier sous l'effet de la température et de l'humidité. Dans notre étude, nous avons vu en effet que le pollen de pelotes perd plus rapidement sa faculté germinative à $+15^{\circ} \mathrm{C}$ qu'à $+7^{\circ} \mathrm{C}$. Mais, à température égale, des variations s'observent aussi selon l'espèce végétale considérée. Ainsi, par exemple, on remarque, à $+7^{\circ} \mathrm{C}$, que le pollen de pelotes de colza qui accuse très tôt un effet d'inhibition a cessé toute activité germinative en moins de 4 jours, tandis qu'il reste encore, d'après Kremer (1949), une petite quantité de pollen de pelotes (de l'ordre de $2 \%$ ) apte à germer au $25^{e}$ jour sur celui du pommier.

A $-20^{\circ} \mathrm{C}$, chez le pommier et le poirier, Klug (1984) note, de son côté, une perte de la valeur germinative du pollen de pelotes de l'ordre de $30 \%$ en 15 jours par rapport à celui de fleurs. Sur le pollen de pelotes prises directement dans les pièges à pollen des ruches, Johansen (1955) avait observé une perte de $62 \%$ de sa vitalité en $5 \mathrm{~h}$ pour le pommier.

Il est vrai que le pollen de colza récolté à la main directement sur les fleurs conserve également moins longtemps sa faculté germinative que celui du pommier. Alors que le pollen de colza semble ces- ser toute activité germinative au $4^{e}$ jour de sa récolte (Mesquida et al., 1987), Okada et al. (1983) obtiennent, dans ce même temps, des taux de germination encore très proches de ceux du jer jour (en tout cas $>50 \%$ ) avec le pollen de pommier, de poirier et de pêcher.

Pour une plus longue durée de conservation de l'aptitude à germer du pollen à basses températures (de l'ordre de $+4^{\circ} \mathrm{C}$ à $-25^{\circ} \mathrm{C}$ ), des techniques ont été adoptées par différents auteurs (Griggs et Vansell, 1949; Johansen, 1955; Dumas, 1982; Filiti et Montalti, 1982; Sakai et al., 1983; Okada et al., 1983; Cerceau-Larrival et Challe, 1986). Elles sont basées essentiellement sur une déshydratation préalable. Or les pollens d'abeilles, de pelotes et de fleurs, utilisés dans nos essais n'ont pas subi de traitement préalable qui aurait été de nature à modifier leur activité germinative. C'est d'ailleurs ce qui explique l'absence de germination des pollens mis à $-25^{\circ} \mathrm{C}$ notamment et aussi à $+30^{\circ} \mathrm{C}$ dans nos conditions expérimentales. Car on sait qu'en appliquant cette technique de la déshydratation, avec du pollen de pommier, Johansen (1955), par exemple, a obtenu un bon taux de germination après un stockage des pelotes de pollen de plusieurs mois à $-18^{\circ} \mathrm{C}$. On sait aussi que le pollen de pommier également (var. «Golden delicious") cueilli à la main sur les fleurs et stocké au froid (à $-25^{\circ} \mathrm{C}$ ) dans un dessicateur conserve une bonne viabilité $(60 \%)$ durant 3 années selon Filiti et Montalti (1982).

Lorsque le pollen de pelotes, pour le cas du pommier, est préalablement déshydraté et rincé avec des solutions de saccharose, puis stocké à basse température $\left(a ̀-10^{\circ} \mathrm{C}\right)$, son aptitude à germer est suffisamment bien conservée pour obtenir, un an après, une efficacité pollinisatrice manuelle de $90 \%$ des fleurs (Okada et al., 1981). 
Incidence sur la pollinisation

Un point reste à discuter : celui de savoir si l'action inhibitrice que les abeilles exercent sur la germination observée in vitro du pollen n'agit pas aussi en facteur limitant de la pollinisation des lignées de colza mâles stériles utilisées en production de semences hybrides F1.

Les résultats obtenus dans ce travail ne permettent pas d'apporter une réponse à ce problème. Cependant, dans une étude antérieure (Mesquida et Renard, 1984), nous avons montré qu'en plein champ les fleurs de colza se trouvaient toujours en conditions de pollinisation saturante. C'est-à-dire que les stigmates des fleurs visitées par les abeilles étaient toujours entièrement, ou presque, recouverts de pollen. Nous avons montré aussi, qu'il suffit d'une couverture aux trois quarts de la surface du stigmate avec du pollen (soit plusieurs centaines) pour obtenir une fécondation satisfaisante des ovules (une trentaine environ). Ainsi, nous pensons que, même avec un taux de germination réduit par effet d'inhibition des sécrétions glandulaires des abeilles (de l'ordre de $40 \%$ ), celles-ci déposeront toujours sur le stigmate des fleurs une quantité suffisamment importante de pollen apte à germer.

II resterait à vérifier que ces résultats obtenus avec des tests de germination in vitro se retrouvent lors d'études in vivo. En effet, d'après les travaux sur Petunia de Lukoschus et Keularts (1968), la pollinisation des fleurs par l'abeille ne semblerait pas influencée par la substance inhibitrice, puisque l'action "pollicide» des acides gras serait inactivée sur le stigmate.

La perte de l'aptitude à germer du pollen n'aurait donc pas, dans la pratique, l'incidence sur la pollinisation que l'on pourrait craindre du fait de l'action «polli- cide" des sécrétions des abeilles observée in vivo. Cela reste à confirmer pour le colza.

\section{Références}

Cerceau-Larrival M.T. \& Challe J. (1986) Biopalynology and maintenance of germination capacity of stored pollen in some angiosperm families. In : Linnean Society Symposium (Blackmore S. \& Ferguson I.K., eds). Série $\mathrm{n}^{\circ}$ 12, pp. 151-164

Dumas C. (1982) Creation d'une banque de pollens : banque de "spermes" pour les plantes à fleurs. Rapport à la Mission des Biotechnologies

Filiti N. \& Montalti P. (1982) In vitro germination and tube growth of apple pollen as affected by cold storage. Rev. Ortoflorofrutticoltura Ital. 66, 361-368

Firman D.T. (1980) Facts of Controlled Pollinisation. Booklet public by Firman Pollen Co. Inc. Wenatchee

Griggs W.H. \& Vansell G.H. (1949) The use of bee collected pollen in artificial pollination of deciduous fruits. Proc. Am. Soc. Hortic. Sci. 54, 118-124

Hoopingarner R., Degrandi-Hoffman G. \& Pulcer R. (1984) Apple pollination : computer simulation and field studies. C.R. V V Symp. Int. sur la Pollinisation, 21, INRA, Paris, pp. 409413

Johansen C.A. (1955) Bee-collected pollen for artificial pollination of apples. Am. Bee J. 95, 352-353

Klug M. (1984) The germination capacity of bee-collected pollen. C.R. Ve Symp. Int. sur la Pollinisation, 21, INRA, Paris, pp. 423-427

Kraai A. (1962) How long do honey-bees carry germinable pollen on them ? Euphytica 11, 5356

Kremer J.C. (1949) Germination tests of the viability of apple pollen gathered in pellets. Proc. Am. Soc. Hortic. Sci. 53, 153-157

Kruskall W.H. \& Wallis W.A. (1952) Use of ranks in one-criterion variance analysis. J. Am. Stat. Assoc. 47, 583-621

Lavie P. (1960) Les substances antibactériennes dans la colonie d'abeilles (Apis mellifica L.). Ann. Abeille 3, 103-183 et 201-305 
Lespinasse Y. \& Chevreau E. (1984) Utilisation d'un gène marqueur pour la recherche de plantes haploïdes de pommier : comparaison entre pollinisation manuelle et pollinisation par les abeilles. C.R. Ve Symp. Int. sur la Pollinisation, 21, INRA, Paris, pp. 391-396

Lukoschus F.S. \& Keularts J.L.W. (1968) Eine weitere Funktion der Mandibeldrüse der Arbeiterin von Apis mellifica L. Produktion eines die Pollenkeimung hemmenden Stoffes. Z. Bienenforsch. 9, 333-343

Maurizio A. (1960) Pollenkeimung hemmende Stoffe im Körper der Honigbiene. Atti ufficiali, XVIi Cong. Internaz. Apicoltori, Imola, 2, pp. 23-25

Maurizio A. (1968) La récolte et l'emmagasinage du pollen par les abeilles. In : Traité de Biologie de l'Abeille, Vol. 3, Masson, Paris, pp. 168-173

Mesquida J. \& Renard M. (1984) Etude des quantités de pollen déposées sur les stigmates dans différentes conditions de pollinisation; influence sur la production de graines chez le colza d'hiver mâle fertile. C.R. Vo Symp. Int. sur la Pollinisation, 21, INRA, Paris, pp. 351. 356

Mesquida J., Renard M. \& Mesquida B. (1987) Etude préliminaire sur la germination in vitro du pollen de colza (Brassica napus L. var. oleifera
Metzger) et sur l'évolution dans le temps de son aptitude à germer. Agronomie 7, 409-416

Okada 1., Sasaki M. \& Maruyama H. (1981) Fruit set in apples hand-pollinated with bee-collected pollen stored for one year. Honeybee Sci. 2, 63-64 (in Japanese)

Okada I., Sakai T. \& Sasaki M. (1983) Washed and pulverized pollen loads of the Honeybees Apis mellifica $\mathrm{L}$. for artificial pollination of fruit trees. Bull. Fac. Agric. Tamagawa Univ. 23, 1835 (in Japanese)

Roberts I.N., Gaude T.C., Harrod G. \& Dickinson H.G. (1983) Pollen stigma interactions in Brassica-oleracea a new pollen germination medium and its use in elucidating the mechanism of self incompatibility. Theor. Appl. Genet. 65, 231-238

Sakai T., Sasaki M. \& Tanaka K. (1983) Utilization of pollen loads from honeybees for artificial pollination of fruit trees, with special reference to the washing method used and longterm preservation. Honeybee Sci. 4, 81-82 (in Japanese) Singh S. \& Boynton D. (1949) Viability of apple pollen in pollen pellets of honeybees. Proc. Am. Soc. Hortic. Sci. 53, 148-152

Williams I.H. (1984) The concentrations of airborne rape pollen over a crop of oil-seed rape (Brassica napus L.). J. Agric. Sci., Camb. 103, 353-357 